\title{
Retraction Note to: Identification of featured biomarkers in different types of lung cancer with DNA microarray
}

\author{
Chao Zhou ${ }^{1}$ Hao Chen ${ }^{1} \cdot$ Li Han ${ }^{1} \cdot$ An Wang ${ }^{1} \cdot$ Liang-an Chen ${ }^{2}$
}

Published online: 18 August 2015

(C) Springer Science+Business Media Dordrecht 2015

\section{Retraction Note to: Mol Biol Rep (2014) 41:6357-6363 \\ DOI 10.1007/s11033-014-3515-9}

The Publisher and Editor retract this article in accordance with the recommendations of the Committee on Publica tion Ethics (COPE). After a thorough investigation we have strong reason to believe that the peer review process was compromised.

zhouchaoem@hotmail.com

$\triangle$ Liang-an Chen

chenlianganc@hotmail.com

1 Department of Respiratory Medicine, Zhou Pu Hospital, 1500 Zhouyuan Road, Pudong new District, Shanghai 201318,

China

2 Department of Respiratory Medicine, Chinese PLA General Hospital, Beijing 100853, China 